\title{
The matching polytope has exponential extension complexity
}

\author{
Thomas Rothvoss* \\ University of Washington, Seattle
}

\begin{abstract}
A popular method in combinatorial optimization is to express polytopes $P$, which may potentially have exponentially many facets, as solutions of linear programs that use few extra variables to reduce the number of constraints down to a polynomial. After two decades of standstill, recent years have brought amazing progress in showing lower bounds for the so called extension complexity, which for a polytope $P$ denotes the smallest number of inequalities necessary to describe a higher dimensional polytope $Q$ that can be linearly projected on $P$.

However, the central question in this field remained wide open: can the perfect matching polytope be written as an LP with polynomially many constraints?

We answer this question negatively. In fact, the extension complexity of the perfect matching polytope in a complete $n$-node graph is $2^{\Omega(n)}$. By a known reduction this also improves the lower bound on the extension complexity for the TSP polytope from $2^{\Omega(\sqrt{n})}$ to $2^{\Omega(n)}$.
\end{abstract}

\section{Introduction}

Linear programs are at the heart of combinatorial optimization as they allow to model a large class of polynomial time solvable problems such as flows, matchings and matroids. The concept of LP duality lead in many cases to structural insights that in turn lead to specialized polynomial time algorithms. In practice, general LP solvers turn out to be very competitive for many problems, even in cases in which specialized algorithms have better theoretical running time. Hence it is particularly interesting to model problems with as few linear constraints as possible. For example if we consider the convex hull $P_{S T}$ of the characteristic vectors of all spanning trees in a complete $n$-node graph, then this polytope has $2^{\Omega(n)}$ many facets [Edm71. However, one can write $P_{S T}=\{x \mid \exists y:(x, y) \in Q\}$ with a higher dimensional polytope $Q$ with only $O\left(n^{3}\right)$ many inequalities [Mar91. Hence, instead of optimizing a linear function over $P_{S T}$, one can optimize over $Q$. In fact, $Q$ is called a linear extension of $P_{S T}$ and the minimum number of facets of any linear extension is called

${ }^{*}$ Email: rothvoss@uw.edu. Part of the work was done while the author was visiting the University of Waterloo and the rest was done while the author was a PostDoc at the Massachusetts Institute of Technology. Research supported in part by ONR grant N00014-11-1-0053 and by NSF contract 1115849. This is the journal version of the paper with the same title that appeared at STOC'14. 
the extension complexity and it is denoted by $\mathrm{xc}\left(P_{S T}\right)$; in this case $\mathrm{xc}\left(P_{S T}\right) \leq O\left(n^{3}\right)$. If $\mathrm{xc}(P)$ is bounded by a polynomial in $n$, then we say that $P \subseteq \mathbb{R}^{n}$ has a compact formulation.

Other examples of non-trivial compact formulations contain the permutahedron [Goe15], the parity polytope, the matching polytope in planar graphs [Bar93] and more generally the matching polytope in graphs with bounded genus Ger91.

A natural question that emerges is which polytopes do not admit a compact formulation. The first progress was made by Yannakakis YYan91 who showed that any symmetric extended formulation for the matching polytope and the TSP polytope must have exponential size. Conveniently, this allowed to reject a sequence of flawed $\mathbf{P}=\mathbf{N P}$ proofs, which claimed to have (complicated) polynomial size LPs for TSP. It was not clear a priori whether the symmetry condition would be essential, but Kaibel, Pashkovich and Theis [KPT10] showed that for the convex hull of all $\log n$-size matchings, there is a compact asymmetric formulation, but no symmetric one. Using a counting argument the author of this paper was able to prove that a random $0 / 1$ polytope would have extension complexity that is exponential in the dimension [Rot12], but without being able to show this for a concrete polytope. In fact, this existential technique was extended to polygons by Fiorini, Rothvoss and Tiwary [FRT12] and to the SDP extension complexity by Briët, Dadush and Pokutta [BDP13].

The major breakthrough by Fiorini, Massar, Pokutta, Tiwary and de Wolf [FMP ${ }^{+12}$ ] showed that several well studied polytopes, including the correlation polytope and the TSP polytope, have exponential extension complexity. More precisely, they show that the rectangle covering lower bound [Yan91 for the correlation polytope is exponential, for which they use known tools from communication complexity such as Razborov's rectangle corruption lemma [Raz90]. Their method was extended by Braun, Fiorini, Pokutta and Steurer [BFPS12] to show also lower bounds on the size of approximations to the correlation polytope; via a reduction this then implies that any polynomial size LP for the convex hull of all cliques in all $n$-node graphs must have an integrality gap of $n^{1 / 2-\varepsilon}$. This quantity was subsequently improved to $n^{1-\varepsilon}$ by Braverman and Moitra [BM13] using an information theory approach; the same bound was later provided by Braun and Pokutta [BP13] using the framework of common information.

One insight that appeared already in Yan91, FMP $^{+} 12$ is that if a "hard" polytope $P$ is the linear projection of a face of another polytope $P^{\prime}$, then $\operatorname{xc}\left(P^{\prime}\right) \geq \operatorname{xc}(P)$. This way, the "hardness" of the correlation polytope can be translated to many other polytopes using a reduction (in fact, in many cases, the usual NP-hardness reduction can be used); see PV13, AT13, for some examples.

A completely independent line of research was given by Chan, Lee, Raghavendra and Steurer [CLRS13] who use techniques from Fourier analysis to show that for constraint satisfaction problems, known integrality gaps for the Sherali-Adams LP translate to lower bounds for any LP of a certain size. For example they show that no LP of size $n^{O(\log n / \log \log n)}$ can approximate MaxCut better than $2-\varepsilon$. This is particularly interesting as in contrast the gap of a polynomial size SDP relaxation is around 1.13, as was shown by Goemans and Williamson GW95.

However, all those polytopes model NP-hard problems and naturally, no complete description of their facets is known (and no efficiently separable description is possible if $\mathbf{N P} \neq \mathbf{c o N P})$. So what about nicely structured combinatorial polytopes that admit polynomial time algorithms to optimize linear functions? The most prominent example here 
is the perfect matching polytope $P_{P M}$, which is the convex hull of all characteristic vectors of perfect matchings in a complete $n$-node graph $G=(V, E)$. A well-known work of Edmonds [Edm65] shows that apart from requiring non-negativity, the degree-constraints plus the odd-cut inequalities are enough for an inequality description. In other words, we can write

$$
\begin{aligned}
P_{P M}(n) & =\operatorname{conv}\left\{\chi_{M} \in \mathbb{R}^{E} \mid M \subseteq E \text { is a perfect matching }\right\} \\
& =\left\{x \in \mathbb{R}^{E}|x(\delta(v))=1 \forall v \in V ; x(\delta(U)) \geq 1 \forall U \subseteq V:| U \mid \text { odd; } x_{e} \geq 0 \forall e \in E\right\} .
\end{aligned}
$$

Here, $\delta(U)$ denotes the set of edges that have exactly one endpoint in $U$. We omit the number $n$ of nodes if they are clear from the context. Note that there are only $n$ degree constraints and $O\left(n^{2}\right)$ non-negativity constraints, but $2^{\Omega(n)}$ odd set inequalities. Any linear function can be optimized over $P_{P M}$ in strongly polynomial time using Edmonds' algorithm [Edm65]. Moreover, given any point $x \notin P_{P M}$, a violating inequality can be found in polynomial time via the equivalence of optimization and separation or using Gomory-Hu trees, see Padberg and Rao [PR82. There are compact formulations for $P_{P M}$ for special graph classes Ger91] and every active cone of $P_{P M}$ admits a compact formulation [VE03. Moreover, the best known upper bound on the extension complexity in general graphs is poly $(n) \cdot 2^{n / 2}$ [FFGT12], which follows from the fact that $\operatorname{poly}(n) \cdot 2^{n / 2}$ many randomly taken complete bipartite graphs cover all matchings and that the convex hull of the union of polytopes can be described with an extended formulation whose size is basically the sum of the individual number of inequalities [Bal85]. Moreover,

$$
\begin{aligned}
P_{M} & =\operatorname{conv}\left\{\chi_{M} \in \mathbb{R}^{E} \mid M \subseteq E \text { is a matching }\right\} \\
& =\left\{x \in \mathbb{R}^{E}\left|x(\delta(v)) \leq 1 \forall v \in V ; x(E(U)) \leq \frac{|U|-1}{2} \forall U \subseteq V:\right| U \mid \text { odd; } x_{e} \geq 0 \forall e \in E\right\}
\end{aligned}
$$

is the convex hull of all matchings in $G$ (not just the perfect ones). Here, $E(U)$ denotes the edges running inside of $U$. Since $P_{P M}$ is a face of $P_{M}$, we have $\mathrm{xc}\left(P_{P M}\right) \leq \mathrm{xc}\left(P_{M}\right)$. But one can also prove that $\mathrm{xc}\left(P_{M}(n)\right) \leq \mathrm{xc}\left(P_{P M}(2 n)\right) 1$. For a detailed discussion of the matching polytope we refer to the book of Schrijver [Sch03].

\subsection{Our contribution}

Despite of all those nice structural properties we show:

Theorem 1. For all even $n$, the extension complexity of the perfect matching polytope in the complete $n$-node graph is $2^{\Omega(n)}$.

This answers a question that was open at least since the paper of Yannakakis [Yan91. The previously best known lower bound was $\Omega\left(n^{2}\right)$ [FKPT13]. As argued above, this also implies that $\mathrm{xc}\left(P_{M}\right) \geq 2^{\Omega(n)}$.

Yannakakis' paper YYan91 also describes a linear projection from a face of the TSP polytope in an $O(n)$-node graph to the perfect matching polytope in an $n$-node graph. This

\footnotetext{
${ }^{1}$ Simply take a complete graph $G=(V, E)$ on $2 n$ nodes and a subset $U \subseteq V$ of $|U|=n$ nodes. Then if we take all perfect matchings $M$ in $G$, then $M \cap E(U)$ gives all matchings in $U$. This construction implies that $P_{M}(n)$ is the linear projection of a face of $P_{P M}(2 n)$.
} 
immediately implies a lower bound for TSP as well, which improves on the $2^{\Omega(\sqrt{n})}$ bound due to $\left[\mathrm{FMP}^{+} 12\right]$.

Corollary 2. For all $n$, the convex hull $P_{T S P}$ of the characteristic vectors of all Hamiltonian cycles in a complete $n$-node graph has extension complexity $2^{\Omega(n)}$.

Also this bound is tight up to constant factors in the exponent. After the publication of the conference version of this paper, Braun and Pokutta BP14 extended our arguments to show that any polytope $K$ with $P_{M} \subseteq K \subseteq\left(1+\frac{1}{2 n}\right) P_{M}$ must still have extension complexity $2^{\Omega(n)}$, implying that there is no "FPTAS-style" approximate extended formulation for matching. We provide a simple reduction which extends their result over the whole parameter range of $\varepsilon$ (see Section 4). It seems this has not been observed before.

Corollary 3. Let $K$ be a polytope with $P_{M} \subseteq K \subseteq(1+\varepsilon) P_{M}$. Then $\mathrm{xc}(K) \geq 2^{\Omega(\min \{1 / \varepsilon, n\})}$.

\section{Our approach}

Formally, the extension complexity $\mathrm{xc}(P)$ is the smallest number of facets of a (higherdimensional) polyhedron $Q$ such that there is a linear projection $\pi$ with $\pi(Q)=P$. This definition seems to ignore the dimension, but one can always eliminate a non-trivial lineality space from $Q$ and make $Q$ full-dimensional, and then the dimension of $Q$ is bounded by the number of inequalities anyway. Before we continue our discussion of the matching polytope, consider a general polytope $P$ and let $x_{1}, \ldots, x_{v}$ be a list of its vertices. Moreover, let $P=\operatorname{conv}\left\{x_{1}, \ldots, x_{v}\right\}=\left\{x \in \mathbb{R}^{n} \mid A x \leq b\right\}$ be any inequality description, say with $f$ inequalities. A crucial concept in extended formulations is the slack matrix $S \in \mathbb{R}_{\geq 0}^{f \times v}$ which is defined by $S_{i j}=b_{i}-A_{i} x_{j}$. Moreover, the non-negative rank of a matrix is

$$
\mathrm{rk}_{+}(S)=\min \left\{r \mid \exists U \in \mathbb{R}_{\geq 0}^{f \times r}, V \in \mathbb{R}_{\geq 0}^{r \times v}: S=U V\right\} .
$$

Recall that if the non-negativity condition is dropped, we recover the usual rank from linear algebra. The connection between extension complexity and non-negative rank is expressed by the following theorem (we reprove the statement here to be fully self-contained):

Theorem 4 (Yannakakis [Yan91]). Let $P$ be a polytope 2 with vertices $\left\{x_{1}, \ldots, x_{v}\right\}$, inequality description $P=\left\{x \in \mathbb{R}^{n} \mid A x \leq b\right\}$ and corresponding slack matrix $S$. Then $\mathrm{xc}(P)=r k_{+}(S)$.

Proof. Let $A_{1}, \ldots, A_{f}$ be the rows of matrix $A$. We begin with showing that $r:=\operatorname{rk}_{+}(S) \Rightarrow$ $\mathrm{xc}(P) \leq r$.

So, suppose that we have a non-negative factorization $S=U V$ with $U \in \mathbb{R}_{\geq 0}^{f \times r}$ and $V \in \mathbb{R}_{\geq 0}^{r \times v}$. We claim that $Q:=\left\{(x, y) \in \mathbb{R}^{n+r} \mid A x+U y=b ; y \geq \mathbf{0}\right\}$ is a linear extension and the projection $\pi$ with $\pi(x, y)=x$ satisfies that $\pi(Q)=P$; in other words, we claim that $P=\left\{x \in \mathbb{R}^{n} \mid \exists y \in \mathbb{R}_{>0}^{r}: A x+U y=b\right\}$. To see this, take a vertex $x_{j}$ of $P$, then we can choose the witness $y:=V^{j}$ and have $\left(x_{j}, y\right) \in Q$ as $A_{i} x_{j}+U_{i} V^{j}=A_{i} x_{j}+S_{i j}=b_{i}$. On

\footnotetext{
${ }^{2}$ For technical reasons we will always assume that the dimension of $P$ is at least 1 .
} 


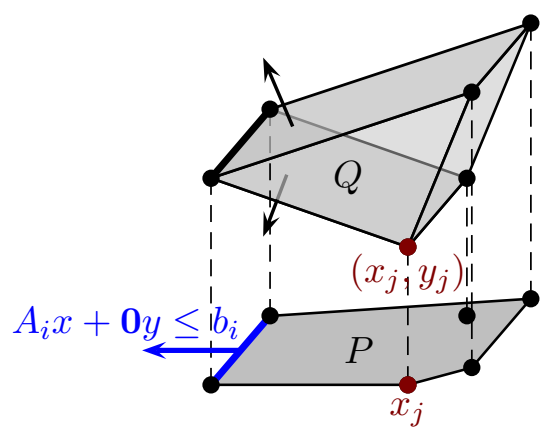

Figure 1: Visualization of Yannakakis' Theorem.

the other hand, if $x \notin P$, then there is some constraint $i$ with $A_{i} x>b_{i}$ and no matter what $y \geq \mathbf{0}$ is chosen, we always have $A_{i} x+U_{i} y \geq A_{i} x>b_{i}$.

For the second part, we have to prove that $r:=\operatorname{xc}(P) \Rightarrow \operatorname{rk}_{+}(S) \leq r$. Hence, suppose that we have a linear extension $Q=\left\{(x, y) \in \mathbb{R}^{n+k} \mid B x+C y \leq d\right\}$ with $r$ inequalities and a linear projection $\pi$ so that $\pi(Q)=P$. After a linear transformation, we may assume that $\pi(x, y)=x$, that means $\pi$ is just the projection on the $x$-variables. We need to come up with vectors $u_{i}, v_{j} \in \mathbb{R}_{>0}^{r}$ so that for each constraint $i$ and each vertex $x_{j}$ one has $\left\langle u_{i}, v_{j}\right\rangle=S_{i j}$. For each point $x_{j}$, fix a lift $\left(x_{j}, y_{j}\right) \in Q$ and choose $v_{j}:=d-B x_{j}-C y_{j} \in \mathbb{R}_{\geq 0}^{r}$ as the vector of slacks that the lift has w.r.t. $Q$. By LP duality we know that each constraint $A_{i} x+\mathbf{0} y \leq b_{i}$ can be derived as a conic combination of the system $B x+C y \leq d$. In other words, there is a vector $u_{i} \in \mathbb{R}_{\geq 0}^{r}$ so that

$$
u_{i}^{T}\left(\begin{array}{l}
B \\
C \\
d
\end{array}\right)=\left(\begin{array}{c}
A_{i} \\
\mathbf{0} \\
b_{i}
\end{array}\right)
$$

Now multiplying gives that

$$
\begin{aligned}
\left\langle u_{i}, v_{j}\right\rangle & =\left\langle u_{i}, d-B x_{j}-C y_{j}\right\rangle \\
& =\underbrace{u_{i}^{T} d}_{=b_{i}}-\underbrace{u_{i}^{T} B}_{=A_{i}} x_{j}-\underbrace{u_{i}^{T} C}_{=\mathbf{0}} y_{j}=b_{i}-A_{i} x_{j}=S_{i j} .
\end{aligned}
$$

In particular, Theorem 4 implies that instead of lower bounding the geometric quantity xc $(P)$ for a polytope $P$ with slack matrix $S$, it fully suffices to find a lower bound for the algebraic quantity $\mathrm{rk}_{+}(S)$. A potential way of lower bounding $\operatorname{rk}_{+}(S)$ was already pointed out in the classical paper of Yannakakis and is known as rectangle covering lower bound: Suppose that $r=\operatorname{rk}_{+}(S)$ and $S=U V$ with $U, V \geq \mathbf{0}$. Then

$$
\operatorname{supp}(S)=\left\{(i, j) \mid S_{i j}>0\right\}=\bigcup_{\ell=1}^{r}\left(\left\{i \mid U_{i \ell}>0\right\} \times\left\{j \mid V_{\ell j}>0\right\}\right)
$$

is a covering of the support of $S$ with $r$ rectangles. In fact, Fiorini et al. [FMP 12$]$ show that the number of rectangles necessary for such a covering of the slack-matrix of the 
correlation polytope is exponential, which in turn lower bounds the extension complexity. More precisely, this is done by considering a cleverly chosen submatrix of the slack-matrix and then applying Razborov [Raz90] as a blackbox.

So, let us discuss the situation for the perfect matching polytope. Since the number of degree constraints and non-negativity inequalities is polynomial anyway, we consider the part of the slack matrix that is induced by the odd set inequalities. In other words, we consider the matrix $S$ with

$$
S_{U M}=|M \cap \delta(U)|-1 \quad \forall M \subseteq E \text { perfect matching } \quad \forall U \subseteq V:|U| \text { odd. }
$$

The first natural approach would be to check whether the rectangle covering lower bound is superpolynomial. Unfortunately, this is not the case, as was already observed in [Yan91. To see this, take any pair $e_{1}, e_{2} \in E$ of non-adjacent edges and choose

$$
\mathcal{M}_{e_{1}, e_{2}}:=\left\{M \mid e_{1}, e_{2} \in M\right\} \quad \text { and } \quad \mathcal{U}_{e_{1}, e_{2}}:=\left\{U \mid e_{1}, e_{2} \in \delta(U)\right\},
$$

then we obtain $O\left(n^{4}\right)$ many rectangles of the form $\mathcal{U}_{e_{1}, e_{2}} \times \mathcal{M}_{e_{1}, e_{2}}$. First of all, we have $S_{U M} \geq\left|\left\{e_{1}, e_{2}\right\}\right|-1 \geq 1$ for each $U \in \mathcal{U}_{e_{1}, e_{2}}$ and $M \in \mathcal{M}_{e_{1}, e_{2}}$, hence the rectangles contain only entries $(U, M)$ that have positive slack. But every entry $(U, M)$ with $S_{U M} \geq 1$ is also contained in at least one such rectangle. To be precise, if $S_{U M}=k$ and $\delta(U) \cap M=$ $\left\{e_{1}, \ldots, e_{k+1}\right\}$, then the entry $(U, M)$ lies in $\left(\begin{array}{c}k+1 \\ 2\end{array}\right)$ rectangles. So the approach with the rectangle covering bound does not work.

On the other hand, considering the rectangle covering as a sum of $O\left(n^{4}\right)$ many $0 / 1$ rank-1 matrices also does not provide a valid non-negative factorization of $S$. The reason is that an entry with $S_{U M}=k$ is contained in $\Theta\left(k^{2}\right)$ many rectangles instead of just $k$ many, thus entries with large slack are over-covered. Moreover, we see no way of rescaling the rectangles in order to fix the problem. This raises the naive question:

Maybe every covering of $S$ with polynomially many rectangles must over-cover entries with large slack?

Surprisingly, it turns out that the answer is "yes"!

To make this more formal, we will use the hyperplane separation lower bound suggested by Fiorini [Fio13]. This bound has been known to experts but has not appeared explicitly in the literature, hence we state it here in generality and include a proof. For matrices $S, W \in \mathbb{R}^{f \times v}$, we will write $\langle S, W\rangle=\sum_{i=1}^{f} \sum_{j=1}^{v} S_{i j} \cdot W_{i j}$ as their Frobenius inner product. Intuitively, the hyperplane separation bound says that if we can find a linear function $W$ that gives a large value for the slack-matrix $S$, but only small values on any rectangle, then the extension complexity is large.

Lemma 5 (Hyperplane separation lower bound [Fio13]). Let $S \in \mathbb{R}_{\geq 0}^{f \times v}$ be the slack-matrix of any polytope $P$ and let $W \in \mathbb{R}^{f \times v}$ be any matrix. Then

$$
x c(P) \geq \frac{\langle W, S\rangle}{\|S\|_{\infty} \cdot \alpha}
$$

with $\alpha:=\max \left\{\langle W, R\rangle \mid R \in\{0,1\}^{f \times v}\right.$ rank-1 matrix $\}$. 
Proof. First, note that the assumption provides that even for any fractional rank-1 matrix $R \in[0,1]^{f \times v}$ one has $\langle W, R\rangle \leq \alpha$. To see this, take an arbitrary rank-1 matrix $R \in[0,1]^{f \times v}$ and write it as $R=x y^{T}$ with vectors $x$ and $y$. After scaling one can assume that $x \in[0,1]^{f}$ and $y \in[0,1]^{v}$. Now suppose that our $R=x y^{T}$ is an optimal solution to

$$
\max \left\{\sum_{i=1}^{f} \sum_{j=1}^{v} W_{i j} \cdot x_{i} y_{j} \mid x_{i}, y_{j} \in[0,1] \forall i \in[f] \forall j \in[v]\right\}
$$

and suppose $R$ is not binary, say because $x \notin\{0,1\}^{v}$. If we fix $y$, this optimization problem is linear in $x$ and there is always an $x^{\prime} \in\{0,1\}^{v}$ that is also optimal. Similarly, also $y$ can be made binary so that the optimum solution to this LP is a rectangle. Geometrically speaking we have just proven that $\operatorname{conv}\left\{R \in[0,1]^{f \times v}: \operatorname{rank}(R) \leq 1\right\}=\operatorname{conv}\left\{R \in\{0,1\}^{f \times v}\right.$ : $\operatorname{rank}(R) \leq 1\}$, even though the set of matrices of rank at most 1 is not a convex set itself.

By Theorem 4 by have $\mathrm{xc}(P)=\mathrm{rk}_{+}(S)$. Now abbreviate $r=\mathrm{rk}_{+}(S)$, then there are $r$ rank-1 matrices $R_{1}, \ldots, R_{r}$ with $S=\sum_{i=1}^{r} R_{i}$. For each $i \in[r]$, we know that $R_{i}$ is non-negative and hence $\frac{1}{\left\|R_{i}\right\|_{\infty}} R_{i}$ is a matrix with entries in $[0,1]$. We obtain

$$
\langle W, S\rangle=\sum_{i=1}^{r}\left\|R_{i}\right\|_{\infty} \cdot \underbrace{\left\langle W, \frac{R_{i}}{\left\|R_{i}\right\|_{\infty}}\right\rangle}_{\leq \alpha} \leq \alpha \cdot \sum_{i=1}^{r} \underbrace{\left\|R_{i}\right\|_{\infty}}_{\leq\|S\|_{\infty}} \leq \alpha \cdot r \cdot\|S\|_{\infty} .
$$

Rearranging gives the claim.

Now, let us go back to the perfect matching polytope and see how we can make use of this bound. Let $k \geq 3$ be an odd integer constant that we choose later. We consider only complete graphs $G=(V, E)$ that have $|V|=n=3 m(k-3)+2 k$ many vertices, for some odd intege 3 . Wherever convenient, we will assume that $n$ and $m$ are large enough, compared to $k$.

Let $\mathcal{M}_{\text {all }}:=\{M \subseteq E \mid M$ is perfect matching $\}$ be the set of all perfect matchings in $G$. We fix $t:=\frac{m+1}{2}(k-3)+3$, which is an odd integer, and consider the set $\mathcal{U}_{\text {all }}:=\{U \subseteq V \mid$ $|U|=t\}$ of all $t$-node cuts in $G$. Let

$$
Q_{\ell}:=\left\{(U, M) \in \mathcal{U}_{\text {all }} \times \mathcal{M}_{\text {all }}|| \delta(U) \cap M \mid=\ell\right\}
$$

be the set of pairs of cuts and matchings intersecting in $\ell$ edges and let $\mu_{\ell}$ be the uniform measure on $Q_{\ell}$. In the following, a rectangle is of the form $\mathcal{R}=\mathcal{U} \times \mathcal{M}$ with $\mathcal{M} \subseteq \mathcal{M}_{\text {all }}$ and $\mathcal{U} \subseteq \mathcal{U}_{\text {all }}$. Note that for parity reasons $\mu_{2 i}(\mathcal{R})=0$ for all $i \in \mathbb{Z}_{\geq 0}$.

Now we want to choose a matrix $W \in \mathbb{R}^{\mathcal{U}_{\text {all }} \times \mathcal{M}_{\text {all }}}$ for which the hyperplane separation bound provides an exponential lower bound. We choose

$$
W_{U, M}= \begin{cases}-\infty & |\delta(U) \cap M|=1 \\ \frac{1}{\left|Q_{3}\right|} & |\delta(U) \cap M|=3 \\ -\frac{1}{k-1} \cdot \frac{1}{\left|Q_{k}\right|} & |\delta(U) \cap M|=k \\ 0 & \text { otherwise. }\end{cases}
$$

\footnotetext{
${ }^{3}$ In other words, we show a lower bound only on $\operatorname{xc}\left(P_{P M}(n)\right)$ for certain $n$. But note that $P_{P M}(n)$ is a face of $P_{P M}\left(n^{\prime}\right)$ for $n \leq n^{\prime}$ and hence $\mathrm{xc}\left(P_{P M}(n)\right) \leq \operatorname{xc}\left(P_{P M}\left(n^{\prime}\right)\right)$. Thus the lower bound on the extension complexity indeed holds for every even $n$.
} 
The intuition is that we reward a rectangle for covering an entry in $Q_{3}$, punish it for covering entries in $Q_{k}$ and completely forbid to cover any entry in $Q_{1}$. First, it is not difficult to see that

$$
\langle W, S\rangle=0+(3-1) \cdot\left|Q_{3}\right| \cdot \frac{1}{\left|Q_{3}\right|}-(k-1) \cdot\left|Q_{k}\right| \cdot \frac{1}{k-1} \cdot \frac{1}{\left|Q_{k}\right|}=1 .
$$

Our hope is that any large rectangle $\mathcal{R}$ must over-cover entries with $|\delta(U) \cap M|=k$ and hence $\langle W, \mathcal{R}\rangle$ is small. In fact, we can prove

Lemma 6. For any large enough odd constant $k$ ( $k:=501$ suffices) and any rectangle $\mathcal{R}$ with $\mathcal{R}=\mathcal{U} \times \mathcal{M}$ with $\mathcal{U} \subseteq \mathcal{U}_{\text {all }}$ and $\mathcal{M} \subseteq \mathcal{M}_{\text {all }}$ one has $\langle W, \mathcal{R}\rangle \leq 2^{-\delta n}$ where $\delta:=\delta(k)>0$ is a constant.

The proof of this lemma is the hard part and takes the complete next section. From the technical point of view, our proof is a substantial modification of Razborov's original rectangle corruption lemma Raz90.

Assuming the bound from Lemma 6 we can then apply Lemma 5, and infer that the perfect matching polytope satisfies

$$
\mathrm{xc}\left(P_{P M}\right) \geq \frac{\langle W, S\rangle}{\|S\|_{\infty} \cdot \max \{\langle W, \mathcal{R}\rangle \mid \mathcal{R} \text { rectangle }\}} \geq \frac{1}{n \cdot 2^{-\delta n}} \geq 2^{\Omega(n)} .
$$

Here we use that $\langle W, S\rangle=1,\|S\|_{\infty} \leq n$ and that $\langle W, \mathcal{R}\rangle \leq 2^{-\delta n}$ for all rectangles $\mathcal{R}$.

\section{The quadratic measure increase}

In this section, we provide the proof of the main technical ingredient, Lemma 6. Formally, we will prove the following statement:

Lemma 7. For each odd $k \geq 3$ and for any rectangle $\mathcal{R}$ with $\mu_{1}(\mathcal{R})=0$, one has $\mu_{3}(\mathcal{R}) \leq$ $\frac{400}{k^{2}} \cdot \mu_{k}(\mathcal{R})+2^{-\delta m}$ where $\delta:=\delta(k)>0$.

We verify that this indeed implies Lemma 6. Consider a rectangle $\mathcal{R}$ and assume that $\mu_{1}(\mathcal{R})=0$ since otherwise $\langle W, \mathcal{R}\rangle=-\infty$. Then

$$
\langle W, \mathcal{R}\rangle=\mu_{3}(\mathcal{R})-\frac{1}{k-1} \mu_{k}(\mathcal{R}) \stackrel{\text { Lem. } 7}{\leq} \underbrace{\left(\frac{400}{k^{2}}-\frac{1}{k-1}\right)}_{\leq 0} \mu_{k}(\mathcal{R})+2^{-\delta m} \leq 2^{-\delta m}
$$

where we choose $k$ as a large enough constant (e.g. $k=501$ ) and recall that $m$ is linear in $n$.

\subsection{The concept of partitions}

The main trick that Razborov used in his classical paper [Raz90] to show a relation between certain measures was to argue that his inequality holds for most random partitions, and that the contribution of the remaining partitions where it does not hold is negligible. In fact, we want to use the same rough idea and translate it to the setting of odd cuts and matchings. 


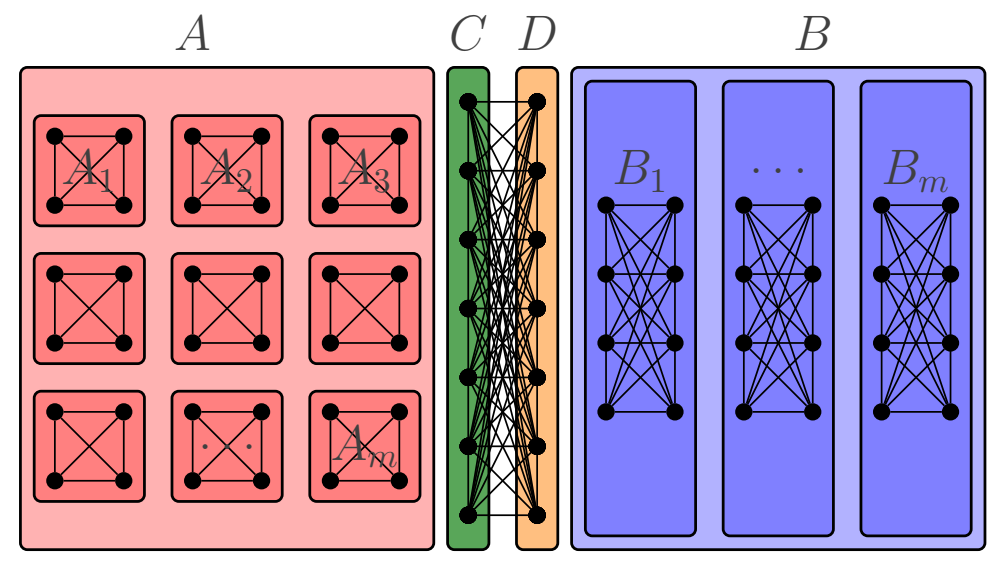

Figure 2: Visualization of a partition $T$ with all edges $E(T)$.

However, our concept of partitions is significantly more involved. For the remainder of Section 3, we fix a rectangle $\mathcal{R}=\mathcal{U} \times \mathcal{M}$ with $\mu_{1}(\mathcal{R})=0$.

A partition is a tuple $T=\left(A=A_{1} \dot{\cup} \ldots \dot{\cup} A_{m}, C, D, B=B_{1} \dot{\cup} \ldots \dot{\cup} B_{m}\right)$ with $V=$ $A \dot{\cup} C \dot{\cup} D \dot{\cup} B$ and the following properties:

- $A \subseteq V$ is a set of $|A|=m(k-3)$ nodes that is partitioned into blocks $A=A_{1} \cup \dot{\cup} \ldots \dot{\cup} A_{m}$ with $\left|A_{i}\right|=k-3$ nodes each.

- $C \subseteq V$ is a set of $k$ nodes.

- $D \subseteq V$ is a set of $k$ nodes.

- $B=B_{1} \dot{\cup} \ldots \dot{\cup} B_{m}$ with $B \subseteq V$ is a partition of the remaining nodes so that $\left|B_{i}\right|=$ $2(k-3)$.

Here, the symbol "Ü" indicates a union of disjoint set. For a node-set $U$, let $E(U):=$ $\{(u, v) \in E \mid u, v \in U\}$ be the edges lying inside of $U$. We abbreviate $E(T):=\bigcup_{i=1}^{m} E\left(A_{i}\right) \cup$ $E(C \cup D) \cup \bigcup_{i=1}^{m} E\left(B_{i}\right)$ as the edges associated with the partition $T$, see Figure 2 ,

We say that

$$
\mathcal{M}_{\text {all }}(T):=\left\{M \in \mathcal{M}_{\text {all }} \mid M \subseteq E(T)\right\}
$$

are all perfect matchings that respect the partition $T$. In other words, the matchings in $\mathcal{M}_{\text {all }}(T)$ have only edges running inside $A_{i}$ or $B_{i}$ or inside $C \cup D$. Similarly, we say that

$$
\mathcal{U}(T):=\left\{U \in \mathcal{U}_{\text {all }} \mid U \subseteq A \cup C \text { with }\left|U \cap A_{i}\right| \in\left\{0,\left|A_{i}\right|\right\} \forall i \in[m]\right\}
$$

are all the $t$-node cuts that respect the partition (see Figure 3). In other words, those cuts are fully contained in $A \cup C$ and for each $i$, they contain either all or none of the nodes $A_{i}$. Moreover, let

$$
\mathcal{M}(T):=\mathcal{M} \cap \mathcal{M}_{\text {all }}(T)=\{M \in \mathcal{M} \mid M \subseteq E(T)\}
$$

and

$$
\mathcal{U}(T):=\mathcal{U} \cap \mathcal{U}_{\text {all }}(T)=\left\{U \in \mathcal{U}|U \subseteq A \cup C ;| U \cap A_{i} \mid \in\left\{0,\left|A_{i}\right|\right\} \forall i \in[m]\right\}
$$




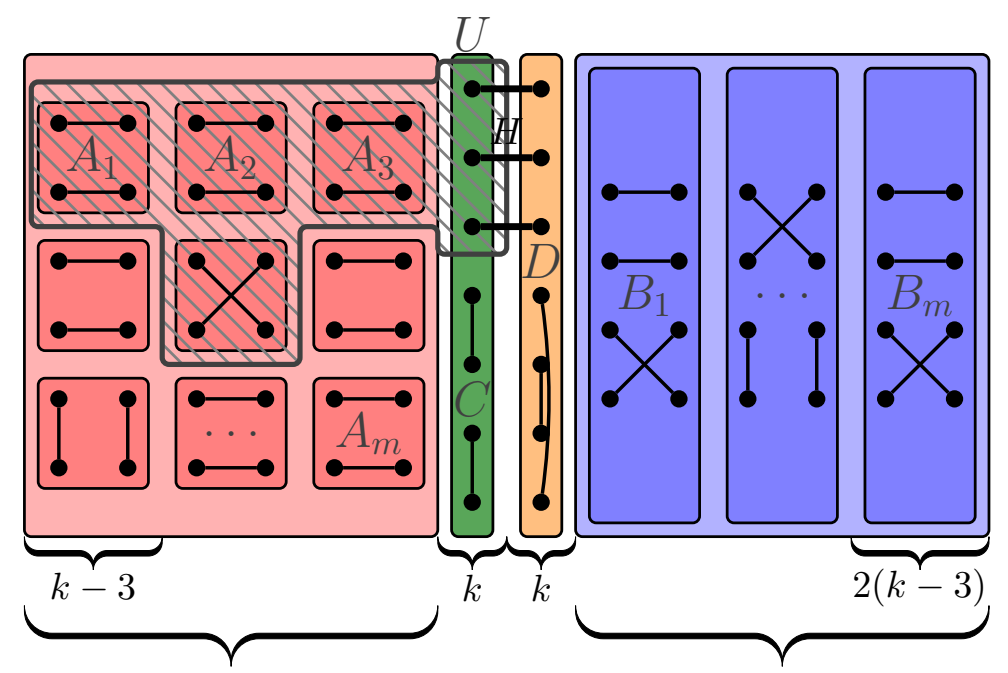

$m$ blocks, each $k-3$ nodes

$m$ blocks, each $2(k-3)$ nodes

$|A|=m(k-3)$ nodes

$|B|=m \cdot 2(k-3)$ nodes

Figure 3: Visualization of a partition $T$ together with one matching $M \in \mathcal{M}(T)$ and one cut $U \in \mathcal{U}(T)$.

be the subsets containing all matchings and cuts from our rectangle $\mathcal{R}$ that respect the partition. The advantage of such partitions $T$ is that if we take a matching $M \in \mathcal{M}_{\text {all }}(T)$ and a cut $U \in \mathcal{U}_{\text {all }}(T)$, then the intersection $\delta(U) \cap M$ can only contain edges in $E(C \cup D)$ (in fact, it contains an odd number between 1 and $k$ edges).

\subsection{Generating the distributions $\mu_{3}$ and $\mu_{k}$}

The key trick is that the measures $\mu_{3}(\mathcal{R})$ and $\mu_{k}(\mathcal{R})$ can be nicely compared for the rectangles $\mathcal{U}(T) \times \mathcal{M}(T)$ that are induced by each partition $T$. Hence, we consider an alternative way to generate uniform members of $Q_{3}$ and $Q_{k}$. To fix some notation, we say that $H$ is an $\ell$-matching if $H$ is a matching with exactly $\ell$ edges. The nodes incident to edges $H$ are denoted by $V(H)$.

For a matching $H \subseteq E(C \cup D)$, we define $p_{\mathcal{M}, T}(H):=\operatorname{Pr}_{M \sim \mathcal{M}_{\text {all }}(T)}[M \in \mathcal{M} \mid H \subseteq M]$ as the chance that a random extension of $H$ to a perfect matching respecting the partition lies in the rectangle. Here, $M \sim \mathcal{M}_{\text {all }}(T)$ indicates that $M$ is a uniformly drawn matching from $\mathcal{M}_{\text {all }}(T)$. If $H \subseteq C \times D$, then we also define $p_{\mathcal{M}, T}^{\text {ex }}(H):=\operatorname{Pr}_{M \sim \mathcal{M}_{\text {all }}(T)}[M \in \mathcal{M} \mid$ $M \cap(C \times D)=H]$ as the probability given that $H$ is the exclusive set of edges that runs between $C$ and $D$. For $c \subseteq C$, let $p_{\mathcal{U}, T}(c):=\operatorname{Pr}_{U \sim \mathcal{U}_{\text {all }}(T)}[U \in \mathcal{U} \mid c \subseteq U]$ be the chance that a random extension of $c$ to a cut $U$ respecting the partition $T$ lies in the rectangle. Again, we also define $p_{\mathcal{U}, T}^{\mathrm{ex}}(c):=\operatorname{Pr}_{U \sim \mathcal{U}_{\text {all }}(T)}[U \in \mathcal{U} \mid U \cap C=c]$ as the chance, given that $c$ are the only nodes in $C$. By a slight abuse of notation, we denote $p_{\mathcal{U}, T}^{\mathrm{ex}}(H):=p_{\mathcal{U}, T}^{\mathrm{ex}}(V(H) \cap C)$ for a matching $H \subseteq C \times D$. Recall that all cuts $U \in \mathcal{U}_{\text {all }}$ have size $|U|=t$; this implies that $|U|-3$ is a multiple of $k-3$, and hence $p_{\mathcal{U}, T}(c)>0$ only if $|c| \in\{3, k\}$.

For the sake of a clearer notation, in the following we will use the symbol $H$ always for 
a 3-matching $H \subseteq C \times D$ and the symbol $F$ will always be used for a $k$-matching that we take either as $F \subseteq E(C \cup D)$ or as $F \subseteq C \times D$. In the remainder of this paper, whenever we write $\mathbb{E}_{T}[\ldots]$, then $T$ is a uniform random partition and if we write $\mathbb{E}_{|H|=3}[\ldots]$, then $H$ is a uniformly picked 3-matching in the complete bipartite graph between $C$ and $D$ (always assuming that the partition $T$ has been selected before). The concept of partitions can be used to generate uniform entries from $Q_{3}$ and $Q_{k}$ in the following way:

- Generating a uniform random entry $(U, M) \in Q_{3}$ : Pick a random partition $T$. Pick a random 3-matching $H \subseteq C \times D$. Then randomly extend $H$ to a matching $M \in \mathcal{M}_{\text {all }}(T)$ with $M \cap(C \times D)=H$ and to a cut $U \in \mathcal{U}_{\text {all }}(T)$ with $\delta(U) \cap(C \times D)=H$. Hence

$$
\mu_{3}(\mathcal{R})=\underset{T}{\mathbb{E}}\left[\underset{|H|=3}{\mathbb{E}}\left[p_{\mathcal{M}, T}^{\mathrm{ex}}(H) \cdot p_{\mathcal{U}, T}^{\mathrm{ex}}(H)\right]\right]
$$

See again Figure 3 ,

- Generating a uniform random entry $(U, M) \in Q_{k}$ : Pick a random partition $T$. Pick a random $k$-matching $F$ in the bipartite graph $C \times D$. Then randomly extend $F$ to a matching $M \in \mathcal{M}_{\text {all }}(T)$ with $M \cap(C \times D)=F$ and to a cut $U \in \mathcal{U}_{\text {all }}(T)$ with $\delta(U) \cap(C \times D)=F$. Hence

$$
\mu_{k}(\mathcal{R})=\underset{T}{\mathbb{E}}\left[\underset{|F|=k}{\mathbb{E}}\left[p_{\mathcal{M}, T}^{\mathrm{ex}}(F) \cdot p_{\mathcal{U}, T}^{\mathrm{ex}}(F)\right]\right]
$$

Note that for a $k$-matching $F \subseteq C \times D$, we anyway have $p_{\mathcal{M}, T}(F)=p_{\mathcal{M}, T}^{\text {ex }}(F)$ and $p_{\mathcal{U}, T}(F)=$ $p_{\mathcal{U}, T}^{\mathrm{ex}}(F)$.

\subsection{The notion of good pairs}

An important definition is the one of good pairs, which are those pairs $(T, H)$ for which we can easily show that their contribution to $\mu_{3}(\mathcal{R})$ is only a $O\left(\frac{1}{k^{2}}\right)$-fraction of the contribution to $\mu_{k}(\mathcal{R})$. In the following, $\varepsilon>0$ denotes a small enough constant that we determine later (in fact $\varepsilon=\frac{1}{8}$ will suffice).

Definition 1 (M-good). Let $T$ be a partition and $H \subseteq C \times D$ be a 3-matching. The pair $(T, H)$ is called $\mathcal{M}$-good if $0<\frac{1}{1+\varepsilon} p_{\mathcal{M}, T}(H) \leq p_{\mathcal{M}, T}(F) \leq(1+\varepsilon) p_{\mathcal{M}, T}(H)$ for all $k$-matchings $F$ with $H \subseteq F \subseteq E(C \cup D)$.

While we prefer this as a formal definition, there is a more intuitive one: imagine we draw a random matching $M$ from $\left\{M^{\prime} \in \mathcal{M}(T) \mid H \subseteq M^{\prime}\right\}$. If $(T, H)$ is $\mathcal{M}$-good, then the induced random matching $M \cap E((C \cup D) \backslash V(H))$ is $\varepsilon$-clos 5 to the distribution of a uniform random matching on the node-set $(C \cup D) \backslash V(H)$. The arguments based on conditional probability can be seen in Cor. 11 in Section 3.5. In particular this will imply

\footnotetext{
${ }^{4}$ We should also argue that $(U, M)$ is indeed a uniform random element from $Q_{3}$. First, note that by construction always $(U, M) \in Q_{3}$. Secondly, $Q_{3}$ is symmetric under permuting nodes. Also the produced pair $(U, M)$ is symmetric under permuting nodes. That gives the claim.

${ }^{5}$ We will not further use the notion of $\varepsilon$-closeness. However, a proper definition would be to call two distributions $\varepsilon$-close if all atomic events have the same probability up to a multiplicative $1+\varepsilon$ factor.
} 
that for a good pair $(T, H)$, all edges in $E((C \cup D) \backslash V(H))$ are contained in at least one matching in $\mathcal{M}(T)$. We have only used $p_{\mathcal{M}, T}$ in the definition of $\mathcal{M}$-goodness and not $p_{\mathcal{M}, T}^{\mathrm{ex}}$. But this is easy to extend:

Lemma 8. Let $T$ be a partition and $H \subseteq C \times D$ be a 3-matching. If ( $T, H)$ is $\mathcal{M}$-good, then $\frac{1}{1+\varepsilon} p_{\mathcal{M}, T}(H) \leq p_{\mathcal{M}, T}^{\mathrm{ex}}(H) \leq(1+\varepsilon) p_{\mathcal{M}, T}(H)$.

Proof. Fix $H$ and take a uniform random $k$-matching $F \sim\left\{F^{\prime} \subseteq E(C \cup D)|| F^{\prime} \mid=\right.$ $k$ and $\left.(C \times D) \cap F^{\prime}=H\right\}$. Then we can write $p_{\mathcal{M}, T}^{\mathrm{ex}}(H)=\mathbb{E}_{F}\left[p_{\mathcal{M}, T}(F)\right] \leq(1+\varepsilon) \cdot p_{\mathcal{M}, T}(H)$ (analogously for the lower bound).

Definition $2(\mathcal{U}$-good). Let $T$ be a partition and $H \subseteq C \times D$ be a 3 -matching. The pair $(T, H)$ is called $\mathcal{U}$-good if $0<\frac{1}{1+\varepsilon} p_{\mathcal{U}, T}^{\mathrm{ex}}(H) \leq p_{\mathcal{U}, T}^{\mathrm{ex}}(C) \leq(1+\varepsilon) p_{\mathcal{U}, T}^{\mathrm{ex}}(H)$.

Again, an alternative characterization for $(T, H)$ with $c=V(H) \cap C$ being $\mathcal{U}$-good is the following: if we draw a random cut $U \sim\left\{U^{\prime} \in \mathcal{U}(T) \mid c \subseteq U^{\prime}\right\}$, then a $\left(\frac{1}{2} \pm \Theta(\varepsilon)\right)$-fraction has $U \cap C=c$ and the other $\left(\frac{1}{2} \pm \Theta(\varepsilon)\right.$ )-fraction has $U \cap C=C$ (recall that every cut $U \in \mathcal{U}(T)$ has $|U \cap C| \in\{3, k\})$. Once more, this characterization can be derived using conditional probabilities, see Cor. 12 .

If $(T, H)$ is both $\mathcal{M}$-good and $\mathcal{U}$-good, then it is called good. There is another type of pairs that will not cause any problems for our analysis: If either $p_{\mathcal{M}, T}^{\mathrm{ex}}(H) \leq 2^{-\delta m}$ or $p_{\mathcal{U}, T}^{\mathrm{ex}}(H) \leq 2^{-\delta m}$, then we say that $(T, H)$ is small. Intuitively, small pairs will only contribute $2^{-\delta m}$ to $\mu_{3}(\mathcal{R})$ anyway.

Unfortunately, those two categories will not cover all cases. Hence, if a pair $(T, H)$ is neither good nor small, then we call it bad. We will use the $0 / 1$ indicator variables $\operatorname{GOOD}(T, H), \operatorname{SMALL}(T, H)$ and $\operatorname{BAD}(T, H)$ for the corresponding events. This allows to split the measure $\mu_{3}(\mathcal{R})$ into 3 parts

$$
\begin{aligned}
\mu_{3}(\mathcal{R}) & =\underset{T}{\mathbb{E}}\left[\underset{|H|=3}{\mathbb{E}}\left[p_{\mathcal{M}, T}^{\mathrm{ex}}(H) \cdot p_{\mathcal{U}, T}^{\mathrm{ex}}(H)\right]\right] \\
& \leq \underset{T}{\mathbb{E}}\left[\underset{|H|=3}{\mathbb{E}}\left[(\operatorname{GOOD}(T, H)+\operatorname{SMALL}(T, H)+\operatorname{BAD}(T, H)) \cdot p_{\mathcal{M}, T}^{\mathrm{ex}}(H) \cdot p_{\mathcal{U}, T}^{\mathrm{ex}}(H)\right]\right] \\
& \stackrel{(*)}{\leq} \underbrace{\frac{200}{k^{2}} \cdot \mu_{k}(\mathcal{R})}_{\text {for good pairs }}+\underbrace{2^{-\delta m}}_{\text {for small pairs }}+\underbrace{\varepsilon \cdot \mu_{3}(\mathcal{R})}_{\text {for bad pairs }}
\end{aligned}
$$

for some constant $\delta:=\delta(k, \varepsilon)>0$. Rearranging terms and choosing $\varepsilon \leq \frac{1}{2}$ gives the claim of Lemma 7 . We will spend the rest of Section 3 justifying the three inequalities that we used in $(*)$.

The estimate that is easy to see is the one concerning the contribution of small pairs. We have

$$
\underset{T}{\mathbb{E}}\left[\underset{|H|=3}{\mathbb{E}}\left[\operatorname{SMALL}(T, H) \cdot p_{\mathcal{M}, T}^{\mathrm{ex}}(H) \cdot p_{\mathcal{U}, T}^{\mathrm{ex}}(H)\right]\right] \leq 2^{-\delta m}
$$

because each time that $\operatorname{SMALL}(T, H)=1$, we have by definition $p_{\mathcal{M}, T}^{\mathrm{ex}}(H) \cdot p_{\mathcal{U}, T}^{\mathrm{ex}}(H) \leq 2^{-\delta m}$. 


\subsection{Contribution of good pairs}

We continue with bounding the contribution of good pairs, i.e. we will show that

$$
\underset{T}{\mathbb{E}}\left[\underset{|H|=3}{\mathbb{E}}\left[\operatorname{GOOD}(T, H) \cdot p_{\mathcal{M}, T}^{\mathrm{ex}}(H) \cdot p_{\mathcal{U}, T}^{\mathrm{ex}}(H)\right]\right] \leq \frac{200}{k^{2}} \cdot \mu_{k}(\mathcal{R})
$$

for $\varepsilon>0$ small enough. The reason, why one should expect a $\frac{1}{k^{2}}$ term is based on the insight that only a $O\left(\frac{1}{k^{2}}\right)$ fraction of 3-matchings $H$ can actually give a positive contribution to the LHS of (3). The reason is that if we had good pairs $(T, H)$ and $\left(T, H^{*}\right)$ where $H$ and $H^{*}$ do not share 2 edges, then we could find a slack-0 entry in $\mathcal{R}$. In fact, this subsection contains the core arguments, why the matching polytope has no compact LP representation. It is also the part where we make use of the combinatorial properties of matchings and cuts.

Lemma 9. For any partition $T$ and any $k$-matching $F \subseteq C \times D$, one has $\operatorname{Pr}_{H \sim\left(\begin{array}{c}F \\ 3\end{array}\right)}[\operatorname{GOOD}(T, H)=$ $1] \leq \frac{100}{k^{2}}$.

Proof. Consider pairs $(T, H)$ and $\left(T, H^{*}\right)$ with $H, H^{*} \subseteq F$ that are both good. We claim that then $\left|H \cap H^{*}\right| \geq 2$. For the sake of contradiction suppose that $\left|H \cap H^{*}\right| \leq 1$. Then there are distinct nodes $u, v \in V\left(H \backslash H^{*}\right) \cap C$. Now arbitrarily extend $H^{*}$ to a $k$-matching $F^{*}$ with $H^{*} \cup\{(u, v)\} \subseteq F^{*} \subseteq E(C \cup D)$.

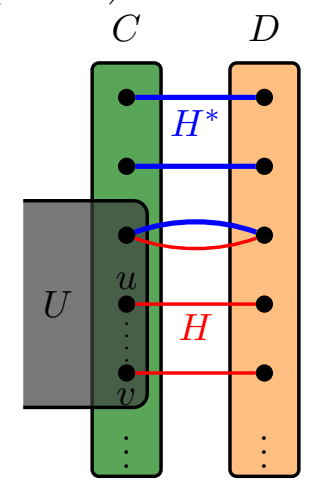

By assumption $\left(T, H^{*}\right)$ is good, hence $p_{\mathcal{M}, T}\left(F^{*}\right) \geq \frac{1}{1+\varepsilon} p_{\mathcal{M}, T}\left(H^{*}\right)>0$. In other words, there exists a matching $M \in \mathcal{M}(T)$ with $(u, v) \in M$. But also $(T, H)$ is good and hence $p_{\mathcal{U}, T}^{\mathrm{ex}}(H)>0$, which implies that there is a cut $U \in \mathcal{U}(T)$ so that $U \cap C=V(H) \cap C$. Then $(u, v)$ runs inside of $U$ and hence $|\delta(U) \cap M|=1$, which is a contradiction to $\mu_{1}(\mathcal{R})=0$. Thus good pairs must indeed overlap in at least 2 edges.

Now fix an $H^{*}$ so that $\left(T, H^{*}\right)$ is good (if there is none, there is nothing to show). Then

$$
\operatorname{Pr}_{H \sim\left(\begin{array}{c}
F \\
3
\end{array}\right)}[\operatorname{GOOD}(T, H)=1] \leq \operatorname{Pr}_{H \sim\left(\begin{array}{c}
F \\
3
\end{array}\right)}\left[\left|H \cap H^{*}\right| \geq 2\right] \leq \frac{3 k}{\left(\begin{array}{c}
k \\
3
\end{array}\right)} \leq \frac{100}{k^{2}} .
$$

This settles the claim.

Now we can easily relate the contribution of the good pairs with the quantity $\mu_{k}(\mathcal{R})$. In particular we use that by definition, for a good pair $(T, H)$ and any $k$-matching $F$ with 
$H \subseteq F \subseteq C \times D$ one has both $p_{\mathcal{M}, T}^{\mathrm{ex}}(H) \leq(1+\varepsilon) p_{\mathcal{M}, T}(H) \leq(1+\varepsilon)^{2} p_{\mathcal{M}, T}^{\mathrm{ex}}(F)$ (see Lemma 8 ) and $p_{\mathcal{U}, T}^{\mathrm{ex}}(H) \leq(1+\varepsilon) p_{\mathcal{U}, T}^{\mathrm{ex}}(C)=(1+\varepsilon) p_{\mathcal{U}, T}^{\mathrm{ex}}(F)$. The inequality in (3) then follows from

$$
\begin{aligned}
& \underset{T}{\mathbb{E}}\left[\underset{|H|=3}{\mathbb{E}}\left[\operatorname{GOOD}(T, H) \cdot p_{\mathcal{M}, T}^{\mathrm{ex}}(H) \cdot p_{\mathcal{U}, T}^{\mathrm{ex}}(H)\right]\right] \\
& =\underset{T}{\mathbb{E}}\left[\underset{|F|=k}{\mathbb{E}}\left[\underset{H \sim\left(\begin{array}{l}
F \\
3
\end{array}\right)}{\mathbb{E}}[\operatorname{GOOD}(T, H) \cdot \underbrace{p_{\mathcal{M}, T}^{\mathrm{ex}}(H)}_{\leq(1+\varepsilon)^{2} p_{\mathcal{M}, T}^{\mathrm{ex}}(F)} \cdot \underbrace{p_{\mathcal{U}, T}^{\mathrm{ex}}(H)}_{\leq(1+\varepsilon) p_{\mathcal{U}, T}^{\mathrm{ex}}(F)}]\right]\right] \\
& \leq 2 \cdot \underbrace{\underset{T}{\mathbb{E}}\left[\underset { | F | = k } { \mathbb { E } } \left[p_{\mathcal{M}, T}^{\mathrm{ex}}(F) \cdot p_{\mathcal{U}, T}^{\mathrm{ex}}(F)\right.\right.}_{=\mu_{k}(\mathcal{R})} \cdot \underbrace{\left.\left.\underset{H \sim\left(\begin{array}{l}
F \\
3
\end{array}\right)}{\mathbb{E}}[\operatorname{GOOD}(T, H)]\right]\right]}_{\leq 100 / k^{2}} \\
& \leq \frac{200}{k^{2}} \cdot \mu_{k}(\mathcal{R})
\end{aligned}
$$

Here we assume that $\varepsilon \leq \frac{1}{8}$.

\subsection{The pseudo-random behavior of large sets}

It remains to bound the contribution of bad pairs. Before we continue with that, we want to describe a general phenomenon concerning the distribution of large set families. To give an example, suppose you have a set family $X \subseteq 2^{[m]}$ with $|X| \geq 2^{(1-\varepsilon) m}$ for a small enough constant $\varepsilon>0$. Then $99 \%$ of indices $i \in\{1, \ldots, m\}$ will be in $50 \% \pm 1 \%$ of sets in the family $X$.

Well, we need a slightly more general statement which we will prove using an entropy counting argument. Recall that for a random variable $y$ over $\{1, \ldots, k\}$, the entropy is defined by $H(y):=\sum_{j=1}^{k} \operatorname{Pr}[y=j] \cdot \log _{2} \frac{1}{\operatorname{Pr}[y=j]}$. Moreover, the entropy is maximized if $y$ is drawn from the uniform distribution; in that case we have $H(y)=\log _{2}(k)$. A useful property is that entropy is sub-additive. For example if $y=\left(y_{1}, \ldots, y_{m}\right)$ is a random vector, then $H(y) \leq \sum_{i=1}^{m} H\left(y_{i}\right)$. In the following, if we write $y \sim Y$, then $y$ is a uniformly drawn random element from $Y$. We need a crucial lemma that appeared already in a less general form in Razborov's paper [Raz90]:

Lemma 10. For all $\varepsilon>0$ and $q \in \mathbb{N}$, there is a constant $\delta:=\delta(\varepsilon, q)>0$ so that the following is true: Take finite sets $X_{1}, \ldots, X_{m}$ with $1 \leq\left|X_{i}\right| \leq q$ for $i=1, \ldots, m$ and denote $X:=X_{1} \times \ldots \times X_{m}$. Let $Y \subseteq X$ be a subset of size $|Y| \geq 2^{-\delta m}|X|$. An index $i \in[m]$ is called $\varepsilon$-unbiased, if

$$
\frac{1}{1+\varepsilon} \cdot \frac{1}{\left|X_{i}\right|} \leq \operatorname{Pr}_{y \sim Y}\left[y_{i}=j\right] \leq(1+\varepsilon) \cdot \frac{1}{\left|X_{i}\right|} \quad \forall j \in X_{i} .
$$

Then at most $\varepsilon m$ many indices will be $\varepsilon$-biased.

Proof. We consider the random variable $y \sim Y$ and fix an index $i$. The entropy of the $i$ th coordinate is

$$
H\left(y_{i}\right)=\sum_{j \in X_{i}} \operatorname{Pr}\left[y_{i}=j\right] \cdot \log _{2}\left(\frac{1}{\operatorname{Pr}\left[y_{i}=j\right]}\right) \leq \log _{2}\left(\left|X_{i}\right|\right)
$$


This bound follows from Jensen's inequality and the observation that the $\log _{2}$ function is concave. In fact, the $\log _{2}$ function is strictly concave, which means that the inequality in (4) is tight only if $\operatorname{Pr}\left[y_{i}=j\right]=\frac{1}{\left|X_{i}\right|}$ for all outcomes $j \in X_{i}$. In particular, if $i$ is biased in the sense of the definition above, then $H\left(y_{i}\right)<\log _{2}\left(\left|X_{i}\right|\right)$. The entropy function $H$ is continuous, hence for compactness reasons there has to be a constant $c:=c(\varepsilon, q)>0$ so that $H\left(y_{i}\right) \leq \log _{2}\left(\left|X_{i}\right|\right)-c$ holds for each $\varepsilon$-biased index $i$.

Now, we assume for the sake of contradiction that there are $\varepsilon m$ indices that are $\varepsilon$-biased. Then we can bound the entropy of $y \sim Y$ by

$$
\log _{2}(|Y|)=H(y) \stackrel{\text { subadd. }}{\leq} \sum_{i=1}^{m} H\left(y_{i}\right) \leq \sum_{i=1}^{m} \log _{2}\left(\left|X_{i}\right|\right)-c \varepsilon m=\log _{2}(|X|)-c \varepsilon m
$$

Rearranging yields $\frac{|Y|}{|X|} \leq 2^{-c \varepsilon m}$, which contradicts the assumption if we choose $\delta<c \varepsilon$.

There is an equivalent way of stating "unbiasedness" that is closer to our definition of good pairs:

Corollary 11. Suppose we have sets $Y \subseteq X=X_{1} \times \ldots \times X_{m}$. For each index $i$ that is $\varepsilon$-unbiased and each $j \in X_{i}$ one has

$$
\frac{1}{1+\varepsilon} \operatorname{Pr}_{y \sim X}[y \in Y] \leq \operatorname{Pr}_{y \sim X}\left[y \in Y \mid y_{i}=j\right] \leq(1+\varepsilon) \operatorname{Pr}_{y \sim X}[y \in Y] .
$$

Proof. We simply rewrite the conditional probability as

$$
\operatorname{Pr}_{y \sim X}\left[y \in Y \mid y_{i}=j\right]=\underbrace{\operatorname{Pr}_{y \sim Y}\left[y_{i}=j\right]}_{\leq(1+\varepsilon) /\left|X_{i}\right|} \cdot \frac{\operatorname{Pr}_{y \sim X}[y \in Y]}{\operatorname{Pr}_{=1 /\left|X_{i}\right|}\left[y_{i}=j\right]} \leq(1+\varepsilon) \cdot \underbrace{\operatorname{Pr}}_{y \sim X}[y \in Y] .
$$

using Bayes' Theorem6. The other direction is analogous.

We will apply Lemma 10 twice in our proof: once for cuts and once for matchings. The cuts all have the same size, so the probability distribution for cuts is not a product distribution. Hence, we need a slight modification:

Corollary 12. Let $Y \subseteq Z \subseteq X=\{0,1\}^{m}$ be sets with $m$ even and $Z:=\left\{y \in\{0,1\}^{m} \mid\right.$ $\left.\|y\|_{1}=\frac{m}{2}\right\}$. Then for each index $i$ that is $\varepsilon$-unbiased with respect to $y \sim Y$ and each $j \in X_{i}$ one has

$$
\frac{1}{1+\varepsilon} \operatorname{Pr}_{y \sim Z}[y \in Y] \leq \operatorname{Pr}_{y \sim Z}\left[y \in Y \mid y_{i}=j\right] \leq(1+\varepsilon) \underset{y \sim Z}{\operatorname{Pr}}[y \in Y]
$$

Proof. We can use the same estimate as in Cor. [11, and observe that still $\operatorname{Pr}_{y \sim Z}\left[y_{i}=j\right]=\frac{1}{2}$ for $j \in\{0,1\}$ even though the uniform distribution over $Z$ is not a product distribution set.

\footnotetext{
${ }^{6}$ Recall that Bayes' Theorem says that $\operatorname{Pr}[A \mid B]=\frac{\operatorname{Pr}[A \cap B]}{\operatorname{Pr}[B]}=\frac{\operatorname{Pr}[A \cap B]}{\operatorname{Pr}[A]} \cdot \frac{\operatorname{Pr}[A]}{\operatorname{Pr}[B]}=\operatorname{Pr}[B \mid A] \cdot \frac{\operatorname{Pr}[A]}{\operatorname{Pr}[B]}$
} 


\subsection{Contribution of bad pairs}

We finally continue with proving that the contribution of the bad pairs is bounded7. Recall that our goal will be to show that

$$
\underset{T}{\mathbb{E}}\left[\underset{|H|=3}{\mathbb{E}}\left[\operatorname{BAD}(T, H) \cdot p_{\mathcal{M}, T}^{\mathrm{ex}}(H) \cdot p_{\mathcal{U}, T}^{\mathrm{ex}}(H)\right]\right] \stackrel{!}{\leq} \varepsilon \cdot \mu_{3}(\mathcal{R}) .
$$

First, observe that the left hand side of (15) can be stated in the following more explicit form

$$
\underset{\substack{T=(A, C, D, B) \\ H \subseteq C \times D:|H|=3}}{\mathbb{E}}\left[\underset{\substack{U \sim \mathcal{U}_{\text {all }}(T): U \cap C=V(H) \cap C \\ M \sim \mathcal{M}_{\text {all }}(T): M \cap(C \times D)=H}}{\mathbb{E}}\left[\operatorname{BAD}(T, H) \cdot \mathbf{1}_{(U, M) \in \mathcal{R}}\right]\right]
$$

Here $1_{\mathcal{E}}$ denotes the indicator variable for an event $\mathcal{E}$. In (6) we pick first the pair $(T, H)$ and then $(U, M)$. Now we want to switch the expectations. For notational convenience, define

$$
\mathcal{P}(U, M):=\left\{\begin{array}{l}
T=(A, C, D, B) \mid \begin{array}{c}
U \in \mathcal{U}_{\text {all }}(T) \text { with } U \cap C=V(H) \cap C \text { and } \\
M \in \mathcal{M}_{\text {all }}(T) \text { with } M \cap(C \times D)=H
\end{array}
\end{array}\right\}
$$

as all the partitions that are compatible with the pair $(U, M) \in Q_{3}$, where $H:=\delta(U) \cap M$. Then switching the expectation gives

$$
(\underline{6})=\underset{\substack{(U, M) \in Q_{3} \\ H:=\delta(U) \cap M}}{\mathbb{E}}[\mathbf{1}_{(U, M) \in \mathcal{R}} \cdot \underbrace{\underset{T \sim \mathcal{P}(U, M)}{\mathbb{E}}[\operatorname{BAD}(T, H)]}_{\text {claim: } \leq \varepsilon}] \stackrel{!}{\leq} \varepsilon \cdot \underset{(U, M) \in Q_{3}}{\mathbb{E}}\left[\mathbf{1}_{(U, M) \in \mathcal{R}}\right]=\varepsilon \cdot \mu_{3}(R) .
$$

Implicitly we have been using here that for symmetry reasons the cardinality $\mathcal{P}(U, M)$ is the same for all $(U, M) \in Q_{3}$. As indicated in the above formula, it suffices to prove that for each pair $(U, M) \in Q_{3}$, only an $\varepsilon$-fraction of compatible partitions $T$ can have the pair $(T, H)$ being bad where $H$ is uniquely defined by $(U, M)$. This then implies (5). Formally, we will prove:

Lemma 13. Fix any $(U, M) \in Q_{3}$ and abbreviate $H:=\delta(U) \cap M$. Then

$$
\underset{T \sim \mathcal{P}(U, M)}{\operatorname{Pr}}[\operatorname{BAD}(T, H)=1] \leq \varepsilon .
$$

Recall that we did call a pair $(T, H)$ bad if it is neither small nor good. Now, let us distinguish whether $(T, H)$ is bad because of the cut part or because of the matching part. We define $(T, H)$ to be $\mathcal{U}$-bad if it is neither small nor $\mathcal{U}$-good. Similarly we call $(T, H)$ $\mathcal{M}$-bad if it is neither small nor $\mathcal{M}$-good. In the next subsections we will separately show that for all $(U, M) \in Q_{3}$ and $H=\delta(U) \cap M$ one has

$$
\underset{T \sim \mathcal{P}(U, M)}{\operatorname{Pr}}[\mathcal{U}-\mathrm{BAD}(T, H)=1] \leq \frac{\varepsilon}{2} \quad \text { and } \quad \underset{T \sim \mathcal{P}(U, M)}{\operatorname{Pr}}[\mathcal{M}-\mathrm{BAD}(T, H)=1] \leq \frac{\varepsilon}{2}
$$

which then concludes Lemma 13 using the union bound.

\footnotetext{
${ }^{7}$ We should mention that the analysis for the bad pairs differs from the STOC'14 version.
} 


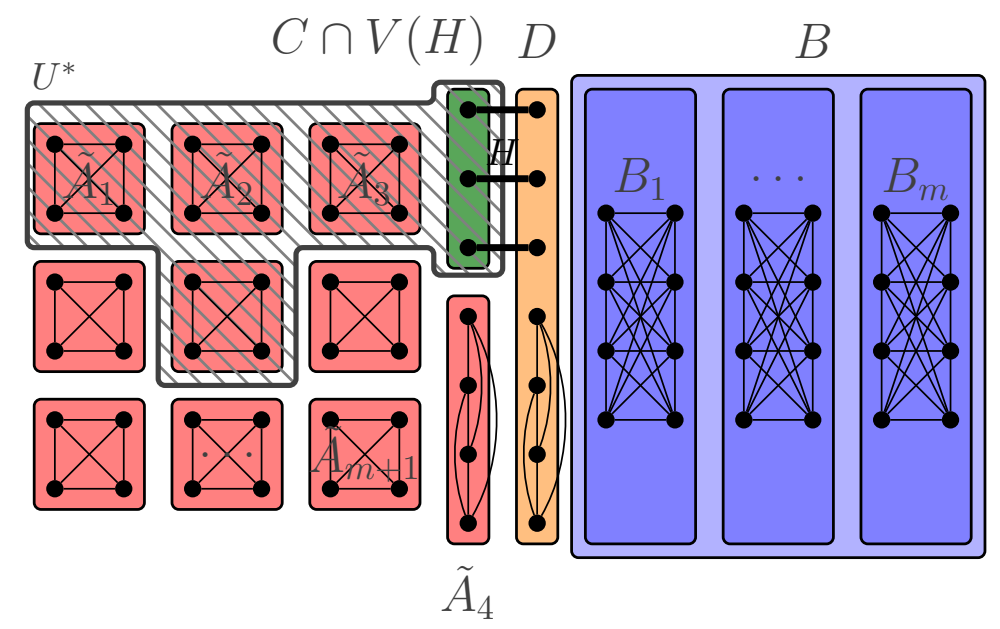

Figure 4: Situation after we decided for $\tilde{A}_{1}, \ldots, \tilde{A}_{m+1}, H, B_{1}, \ldots, B_{m}$. The matching $M^{*}$ is a subset of the depicted edges.

\subsubsection{The contribution for $\mathcal{U}$-bad pairs}

First, we show that for any pair $(U, M)$, most compatible partitions $T$ have either $(T, H)$ being small or $\mathcal{U}$-good. If we have a perfect matching $M$ and a nodeset $W$, then we say that $M$ crosses $W$ if $M \cap \delta(W) \neq \emptyset$.

Lemma 14. Fix any pair $\left(U^{*}, M^{*}\right) \in Q_{3}$ and abbreviate $H:=\delta\left(U^{*}\right) \cap M^{*}$. Then

$$
\underset{T \sim \mathcal{P}\left(U^{*}, M^{*}\right)}{\operatorname{Pr}}[\mathcal{U}-\operatorname{BAD}(T, H)=1] \leq \frac{\varepsilon}{2}
$$

Proof. We imagine that we generate the random partition $T \sim \mathcal{P}\left(U^{*}, M^{*}\right)$ in two phases. In the first phase, we randomly partition the nodes $V \backslash V(H)$ into disjoint blocks $B_{1}, \ldots, B_{m}$ each of size $2(k-3) ; k-3$ nodes $D \backslash V(H)$ and blocks $\tilde{A}_{1}, \ldots, \tilde{A}_{m+1}$, each of size $k-3$. Here we choose the blocks conditioning on the following events: (1) $M^{*}$ does not cross any of those blocks; (2) $U^{*} \subseteq V \backslash(B \cup D)$ and (3) $\left|U^{*} \cap \tilde{A}_{i}\right| \in\left\{0,\left|\tilde{A}_{i}\right|\right\}$ for all $i=1, \ldots, m+1$. Figure 4 gives a visualization.

Now, consider the indices $J:=\left\{i \in[m+1]|| U^{*} \cap \tilde{A}_{i} \mid=0\right\}$ of blocks not contained in $U^{*}$. In particular $|J|=\frac{m+1}{2}$. In the second phase, we take a random uniform index $i \sim J$ and declare $\tilde{A}_{i}$ as the missing $k-3$ nodes $C \backslash V(H)$. Finally, we set $\left\{A_{1}, \ldots, A_{m}\right\}:=$ $\left\{\tilde{A}_{1}, \ldots, \tilde{A}_{i-1}, \tilde{A}_{i+1}, \ldots, \tilde{A}_{m+1}\right\}$ which completes the description of $T$. The two stage process can be summarized to

$$
\operatorname{Pr}_{T \sim \mathcal{P}\left(U^{*}, M^{*}\right)}[\mathcal{U}-\operatorname{BAD}(T, H)=1]=\underset{B_{1}, \ldots, B_{m}, D, \tilde{A}_{1}, \ldots, \tilde{A}_{m+1}}{\mathbb{E}}[\underset{i \sim J}{\mathbb{E}}[\mathcal{U}-\mathrm{BAD}(T, H)]] .
$$

Now fix any outcome of $B_{1}, \ldots, B_{m}, D, \tilde{A}_{1}, \ldots, \tilde{A}_{m+1}$ in the first phase; we will show that in any case $\operatorname{Pr}_{i \sim J}[\mathcal{U}-\operatorname{BAD}(T, H)=1] \leq \frac{\varepsilon}{2}$. For a vector $y \in\{0,1\}^{m+1}$, we define a cut

$$
f(y):=(C \cap V(H)) \cup \bigcup_{i: y_{i}=1} \tilde{A}_{i}
$$


and denote $Y:=\left\{y \in\{0,1\}^{m+1}: f(y) \in \mathcal{U}\right\}$ as well as $Z:=\left\{y \in\{0,1\}^{m+1}:\|y\|_{1}=\frac{m+1}{2}\right\}$ and $X:=\{0,1\}^{m+1}$. In other words, the vectors in $Y$ represent all the cuts in the rectangle $\mathcal{R}$ that determine the outcome of $\mathcal{U}-\operatorname{BAD}(T, H)$ in the second phase. More precisely, we know that a partition $(T, H)$ will be $\mathcal{U}$-good, if in the second phase we pick an index $i$ so that roughly half of the vectors $y \sim Y$ have $y_{i}=1$. Our goal is to use the insight from Section 3.5 to argue that this is the case for most indices. We can assume that there is at least one index $i^{*} \in J$ so that the outcome $(T, H)$ is not small, since otherwise there is nothing to show. The partition for that index satisfies $p_{\mathcal{U}, T}^{\mathrm{ex}}(H) \geq 2^{-\delta m}$ and hence $|Y| \geq 2^{-\delta m}\left(\begin{array}{c}m \\ (m+1) / 2\end{array}\right) \geq 2^{-2 \delta m}|X|$ for $m$ large enough. Now we apply Lemma 10 for a small enough $\delta>0$ and obtain that at most $\frac{\varepsilon}{4} m$ many indices are $\varepsilon$-biased. Then the chance of picking a bad index is at most $\frac{\varepsilon m / 4}{|J|} \leq \frac{\varepsilon}{2}$. Finally, we apply Cor. 12 to conclude that for a $\left(1-\frac{\varepsilon}{2}\right)$-fraction of indices, the corresponding partitions are $\mathcal{U}$-good.

Admittedly, only now one can fully understand the meaning behind the definition of a partition: if we take a partition $\left(T^{\prime}, H\right)$, then the set $C \backslash V(H)$ contains the same number of nodes as any $A_{i}$. Assuming that there are enough cuts in $\mathcal{U}^{\prime}:=\left\{U \in \mathcal{U}\left(T^{\prime}\right) \mid(V(H) \cap C) \subseteq\right.$ $U$ \}, then we know that for most indices $i$, roughly half the cuts of $\mathcal{U}^{\prime}$ will contain $A_{i}$ - the other half will not. Hence one could imagine to randomly pick an index $i$ and exchange the set $C \backslash V(H)$ with $A_{i}$ and the emerging pair $(T, H)$ would then be $\mathcal{U}$-good with probability $1-\frac{\varepsilon}{2}$. This is a slightly different view on the argument in the proof of Lemma 14.

\subsubsection{The contribution for $\mathcal{M}$-bad pairs}

Bounding the chance that a pair $(T, H)$ turns out to be $\mathcal{M}$-bad is fairly similar to the previous case. Now it will be crucial that $(C \cup D) \backslash V(H)$ has the same size as each block $B_{i}$ so that we can again play the exchange trick.

Lemma 15. Fix $\left(U^{*}, M^{*}\right) \in Q_{3}$ and let $H:=\delta\left(U^{*}\right) \cap M^{*}$. Then

$$
\underset{T \sim \mathcal{P}\left(U^{*}, M^{*}\right)}{\operatorname{Pr}}[\mathcal{M}-\operatorname{BAD}(T, H)=1] \leq \frac{\varepsilon}{2} .
$$

Proof. Again, we imagine that we draw the partition $T$ in two phase. In the first phase, we pick blocks $\tilde{B}_{1}, \ldots, \tilde{B}_{m+1}$ of each $2(k-3)$ nodes disjoint to $U^{*}$. Then we select blocks $A_{1}, \ldots, A_{m}$ of $k-3$ nodes each. Again we condition that $M^{*}$ is not crossing any of those blocks $\tilde{B}_{i}$ or $A_{i}$. Then we randomly partition each $\tilde{B}_{i}$-block into $\tilde{B}_{i}=C_{i} \cup \dot{\cup} D_{i}$ with $\left|C_{i}\right|=$ $\left|D_{i}\right|=k-3$ conditioning that $M^{*} \cap\left(C_{i} \times D_{i}\right)=\emptyset$ for all $i=1, \ldots, m+1$. Figure 5 shows that situation. Note that at this point, we have only determined 3 nodes in each $C$ and $D$. In the second phase, we select a uniform random index $i \in[m+1]$ and set $C:=(C \cap V(H)) \cup C_{i}$ and $D:=(V(H) \cap D) \cup D_{i}$. Eventually, we set $\left\{B_{1}, \ldots, B_{m}\right\}:=$ $\left\{\tilde{B}_{1}, \ldots, \tilde{B}_{i-1}, \tilde{B}_{i+1}, \ldots, \tilde{B}_{m+1}\right\}$ which again completes the description of the partition $T$. Observe that for symmetry reasons, the generated partition $T$ is a uniform element of $\mathcal{P}\left(U^{*}, M^{*}\right)$.

As before, we fix any outcome in the first phase and claim that still $\operatorname{Pr}_{i \in[m+1]}[\mathcal{M}-\operatorname{BAD}(T, H)=$ 


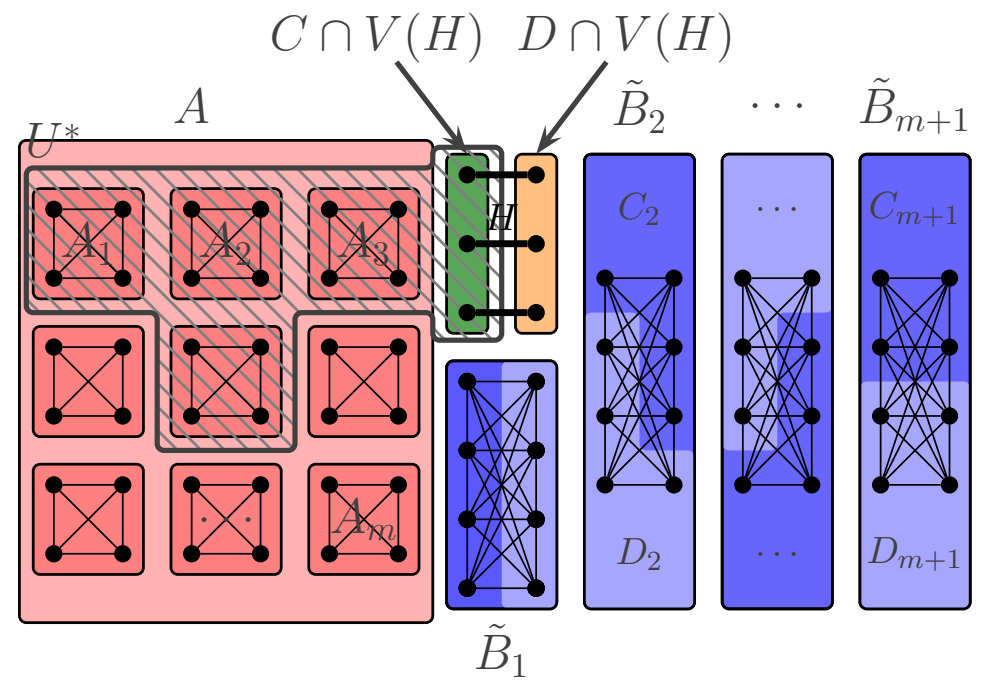

Figure 5: Situation after we decided for $A_{1}, \ldots, A_{m}, H, \tilde{B}_{1}, \ldots, \tilde{B}_{m+1}$ and $\tilde{B}_{i}=C_{i} \dot{\cup} D_{i}$. Again, $M^{*}$ is a subset of the depicted edges.

$1] \leq \frac{\varepsilon}{2}$. Define

$$
\begin{aligned}
X_{i} & :=\left\{\text { all perfect matchings on } \tilde{B}_{i}\right\} & \forall i=1, \ldots, m+1 \\
X_{m+i+1} & :=\left\{\text { all perfect matchings on } A_{i}\right\} & \forall i=1, \ldots, m
\end{aligned}
$$

and set $X:=X_{1} \times \ldots \times X_{2 m+1}$. We abbreviate $Y:=\{M \in X \mid M \cup H \in \mathcal{M}\}$ as the corresponding matchings that are in our rectangle $\mathcal{R}$. Again, we can assume that for some index $i$, the pair $(T, H)$ is not small and hence $|Y| \geq 2^{-\delta m} \prod_{j \neq i}\left|X_{j}\right| \geq 2^{-2 \delta m}|X|$ for $m$ large enough.

The definition of $\mathcal{M}$-goodness requires that if we draw $M \sim Y$, then the induced matching that we see on $\tilde{B}_{i}$ is very close to a uniform random matching. Again for a small enough $\delta$, Lemma 10 bounds the number of indices that are $\varepsilon$-biased by $\frac{\varepsilon}{2} m$. Here $q:=\max \left\{\left|X_{i}\right|: i=1, \ldots, 2 m+1\right\}$ is the number of matchings on $2(k-3)$ nodes, which is some (huge) constant depending on $k$. Following Cor. 11, an unbiased index implies that the corresponding pair $(T, H)$ will be $\mathcal{M}$-good. This shows the claim.

This concludes the proof the Lemma 7 and hence implies our main result, Theorem 1.

\section{Inapproximability of the matching polytope}

In this section, we want to discuss the inapproximability for $P_{M}$, which is the convex hull of all matchings in $G$ (not just the perfect ones). The polytope $P_{M}$ has the nice property that it is monotone, which means that for $x \in P_{M}$ and $\mathbf{0} \leq y \leq x$ one also has $y \in P_{M}$. We say that a polytope $K$ is a $(1+\varepsilon)$-approximation to $P_{M}$ if $P_{M} \subseteq K \subseteq(1+\varepsilon) P_{M}$ (note that this only makes sense for monotone polytopes). This is equivalent to requiring that for each objective function $c \in \mathbb{R}_{\geq 0}^{E}$ one has

$$
\max \left\{c x \mid x \in P_{M}\right\} \leq \max \{c x \mid x \in K\} \leq(1+\varepsilon) \cdot \max \left\{c x \mid x \in P_{M}\right\},
$$


where we use again monotonicity.

After the publication of the conference version of this work, Braun and Pokutta [BP14] showed the following extension:

Theorem 16. Let $0<\alpha<1$. If $K$ is a polytope with $P_{M} \subseteq K \subseteq\left(1+\frac{\alpha}{n}\right) P_{M}$, then $\mathrm{xc}(K) \geq 2^{c n}$ with $c:=c(\alpha)>0$ depending on $\alpha$.

However, their paper leaves it open how large the extension complexity of a $(1+\varepsilon)$ approximation has to be if $\varepsilon \geq \frac{1}{n}$. For the sake of comparison, suppose in (11) we would take the odd cut inequalities only for $|U| \leq \Theta\left(\frac{1}{\varepsilon}\right)$, then we would obtain a polytope $K$ with $P_{M} \subseteq K \subseteq(1+\varepsilon) P_{M}$ which has only $n^{\Theta(1 / \varepsilon)}=2^{\Theta\left(\frac{\log n}{\varepsilon}\right)}$ many facet: 8 .

We want to argue now that the result of Braun and Pokutta [BP14] implies a lower bound for the whole spectrum of $\varepsilon$ :

Corollary 17. Let $G=(V, E)$ be the complete graph on $n$ nodes and let $P_{M}$ be the convex hull of all matchings. Let $K$ be a polytope with $P_{M} \subseteq K \subseteq(1+\varepsilon) P_{M}$. Then $x c(K) \geq 2^{\Omega(\min \{1 / \varepsilon, n\})}$.

Proof. Assume that $\varepsilon \geq \frac{1}{2 n}$, otherwise the original result of Braun and Pokutta already applies. We may also assume that $\frac{1}{\varepsilon}$ is an even integer. Let us write $P_{M}(G)$ to emphasize that we talk about the matching polytope for graph $G$. Suppose that we have a polytope $K=\left\{x \in \mathbb{R}^{E}: \exists y:(x, y) \in Q\right\}$ with $P_{M}(G) \subseteq K \subseteq(1+\varepsilon) \cdot P_{M}(G)$ so that $Q$ has $\mathrm{xc}(K)$ many facets. Take any set $V^{\prime} \subseteq V$ of $k:=\frac{1}{2 \varepsilon} \leq n$ vertices and let $G^{\prime}=\left(V^{\prime}, E^{\prime}\right)$ be the induced subgraph. For a vector $x \in \mathbb{R}^{E}$, we write $x=\left(x^{\prime}, x^{\prime \prime}\right)$ with $x^{\prime} \in \mathbb{R}^{E^{\prime}}$ and $x^{\prime \prime} \in \mathbb{R}^{E / E^{\prime}}$. Then

$$
K^{\prime}:=\left\{x^{\prime} \in \mathbb{R}^{E^{\prime}} \mid \exists y:\left(\left(x^{\prime}, \mathbf{0}\right), y\right) \in Q\right\}
$$

is a polytope with $\mathrm{xc}\left(K^{\prime}\right) \leq \mathrm{xc}(K)$. Intuitively, $K^{\prime}$ emerges from $K$ by "deleting" variables for edges that are not in $G^{\prime}$. But $K^{\prime}$ is still an approximation to the matching polytope for $G^{\prime}$; formally $P_{M}\left(G^{\prime}\right) \subseteq K^{\prime} \subseteq(1+\varepsilon) P_{M}\left(G^{\prime}\right)$. This can be easily seen by checking criterion (7) for objective functions $c$ with $c_{e}=0$ for $e \in E \backslash E^{\prime}$. But for this smaller graph, we have that $\varepsilon=\frac{1}{2\left|V^{\prime}\right|}$, hence we can apply the original result of Braun and Pokutta to the graph $G^{\prime}$ and obtain that $\mathrm{xc}\left(K^{\prime}\right) \geq 2^{\Omega\left(\left|V^{\prime}\right|\right)}$. This shows the claim.

This provides a fairly tight bound on the approximability of the matching polytope whenever $\varepsilon \ll \frac{1}{\log n}$. However, the gap between known upper and lower bound remains huge if $\varepsilon$ is a constant.

\subsection{Subsequent development}

After a sequence of papers showed lower bounds on the size of linear programs (including FMP $^{+} 12$, CLRS13 and this paper), the natural next challenge was whether one could also

\footnotetext{
${ }^{8}$ To see that $K \subseteq(1+\varepsilon) P_{M}$, suppose that $x \in \mathbb{R}_{\geq 0}^{E}$ satisfies $x(\delta(v)) \leq 1$ for $v \in V$ and $x(E(U)) \leq \frac{|U|-1}{2}$ for every odd $U \subseteq V$ of size $|U| \leq k$. Already from the degree constraints we know that for any $U$ we have at least $x(E(U)) \leq \frac{|U|}{2}$. If we define a slightly scaled vector $y:=\left(1-\frac{1}{k}\right) \cdot x$, then for any odd $U$ of size $|U|>k$ we have $y(E(U)) \leq\left(1-\frac{1}{k}\right) \frac{|U|}{2} \leq \frac{|U|-1}{2}$. Hence $y \in P_{M}$.
} 
prove lower bounds on the size of semidefinite programs. A recent breakthrough of Lee, Raghavendra and Steurer [LRS15] answers this affirmatively for the correlation polytope, the cut polytope and approximate versions of constraint satisfaction problems. However, it is still unknown whether there is a polynomial size SDP for the perfect matching polytope.

\subsection{Acknowledgements}

The author is grateful to Fritz Eisenbrand, Michel X. Goemans, Jochen Könemann and Laura Sanità for helpful discussions and to Sam Fiorini for reading and commenting on a preliminary draft. Moreover, the author wants to thank the anonymous reviewers of both the conference and the journal version for their numerous helpful suggestions and comments.

\section{References}

[AT13] D. Avis and H. R. Tiwary. On the extension complexity of combinatorial polytopes. In $I C A L P$, pages 57-68, 2013.

[Bal85] E. Balas. Disjunctive programming and a hierarchy of relaxations for discrete optimization problems. SIAM J. Algebraic Discrete Methods, 6(3):466-486, 1985.

[Bar93] F. Barahona. On cuts and matchings in planar graphs. Mathematical Programming, 60:53-68, 1993.

[BDP13] J Briët, D. Dadush, and S. Pokutta. On the existence of 0/1 polytopes with high semidefinite extension complexity. In ESA, pages 217-228, 2013.

[BFPS12] G. Braun, S. Fiorini, S. Pokutta, and D. Steurer. Approximation limits of linear programs (beyond hierarchies). In FOCS, pages 480-489, 2012.

[BM13] M. Braverman and A. Moitra. An information complexity approach to extended formulations. In STOC, pages 161-170, 2013.

[BP13] G. Braun and S. Pokutta. Common information and unique disjointness. Electronic Colloquium on Computational Complexity (ECCC), 20:56, 2013.

[BP14] G. Braun and S. Pokutta. The matching polytope does not admit fullypolynomial size relaxation schemes. CoRR, abs/1403.6710, 2014.

[CLRS13] S. O. Chan, J. R. Lee, P. Raghavendra, and D. Steurer. Approximate constraint satisfaction requires large LP relaxations. In FOCS, pages 350-359, 2013.

[Edm65] J. Edmonds. Maximum matching and a polyhedron with 0,1-vertices. J. Res. Nat. Bur. Standards Sect. B, 69B:125-130, 1965.

[Edm71] J. Edmonds. Matroids and the greedy algorithm. Mathematical Programming, 1(1):127-136, 1971.

[FFGT12] Y. Faenza, S. Fiorini, R. Grappe, and H. R. Tiwary. Extended formulations, nonnegative factorizations, and randomized communication protocols. In ISCO, pages 129-140, 2012. 
[Fio13] S. Fiorini. Personal communication, 2013.

[FKPT13] S. Fiorini, V. Kaibel, K. Pashkovich, and D. O. Theis. Combinatorial bounds on nonnegative rank and extended formulations. Discrete Mathematics, 313(1):6783, 2013.

$\left[\mathrm{FMP}^{+}\right.$12] S. Fiorini, S. Massar, S. Pokutta, H. Tiwary, and R. de Wolf. Linear vs. semidefinite extended formulations: exponential separation and strong lower bounds. In $S T O C$, pages 95-106, 2012.

[FRT12] S. Fiorini, T. Rothvoß, and H. R. Tiwary. Extended formulations for polygons. Discrete $\&$ Computational Geometry, 48(3):658-668, 2012.

[Ger91] A. M. H. Gerards. Compact systems for T-join and perfect matching polyhedra of graphs with bounded genus. Operations Research Letters, 10(7):377 - 382, 1991.

[Goe15] M. X. Goemans. Smallest compact formulation for the permutahedron. Math. Program., 153(1):5-11, 2015.

[GW95] M. X. Goemans and D. P. Williamson. Improved approximation algorithms for maximum cut and satisfiability problems using semidefinite programming. $J$. ACM, 42(6):1115-1145, 1995.

[KPT10] V. Kaibel, K. Pashkovich, and D. O. Theis. Symmetry matters for the sizes of extended formulations. In IPCO, pages 135-148, 2010.

[LRS15] J. R. Lee, P. Raghavendra, and D. Steurer. Lower bounds on the size of semidefinite programming relaxations. In STOC, pages 567-576, 2015.

[Mar91] R. Kipp Martin. Using separation algorithms to generate mixed integer model reformulations. Operations Research Letters, 10(3):119 - 128, 1991.

[PR82] M. Padberg and M. Rao. Odd minimum cut-sets and b-matchings. Mathematics of Operations Research, pages 7:67-80, 1982.

[PV13] S. Pokutta and M. Van Vyve. A note on the extension complexity of the knapsack polytope. Oper. Res. Lett., 41(4):347-350, 2013.

[Raz90] A. Razborov. On the distributional complexity of disjointness. In ICALP, pages 249-253, 1990.

[Rot12] T. Rothvoss. Some 0/1 polytopes need exponential size extended formulations. Mathematical Programming, pages 1-14, 2012.

[Sch03] A. Schrijver. Combinatorial optimization. Polyhedra and efficiency. Vol. A,B,C, volume 24 of Algorithms and Combinatorics. Springer-Verlag, Berlin, 2003.

[VE03] P. Ventura and F. Eisenbrand. A compact linear program for testing optimality of perfect matchings. Oper. Res. Lett., 31(3):429-434, 2003. 
[Yan91] M. Yannakakis. Expressing combinatorial optimization problems by linear programs. Journal of Computer and System Sciences, 43(3):441 - 466, 1991. 